\title{
Neonatal tetanus associated with skin infection
}

\author{
M Maharaj, MB ChB; N Dungwa, MB ChB, DCH, FC Paed (SA), FAAFP, FCFP, Dip HIV Man \\ Department of Paediatrics and Child Health, Witbank Hospital and School of Medicine, Faculty of Health Sciences, University of Pretoria, \\ South Africa
}

Correspondingauthor: N Dungwa (nomdungwa@yahoo.com)

\begin{abstract}
A 1-week-old infant was brought to a regional hospital with a history of recurrent seizures following lower abdominal septic skin infection. She was found to have neonatal tetanus, and a spatula test was positive. The tetanus infection was associated with a superficial skin infection, common in neonates. Treatment included sedatives (diazepam, chlorpromazine, phenobarbitone and morphine), muscle relaxants, antibiotics and ventilation in the neonatal intensive care unit. Intrathecal and intramuscular immunoglobulin were given, and the wound was treated. The infant recovered, with no seizures by the 16 th day from admission, and was off the ventilator by the 18 th day. This was shorter than the usual 3 - 4 weeks for neonates with tetanus at the hospital. The question arises whether tetanus immunisation should be considered in infants with skin infections, which frequently occur in the neonatal period.
\end{abstract}

S Afr Med J 2016;106(9):888-890. DOI:10.7196/SAMJ.2016.v106i9.11139

The World Health Organization (WHO) defines South Africa (SA) as having eliminated neonatal tetanus. The National Department of Health $(\mathrm{NDoH})$ treatment guidelines no longer cover the treatment of severe neonatal tetanus. ${ }^{[1]}$ However, clinicians need to be alert, as cases do arise even in countries considered to have eliminated the disease. We have encountered three clinical cases, including the one presented here, over a period of 8 months. Inadequate antenatal immunisation was a factor in the case presented. We present a protocol for doctors encountering this rare but life-threatening condition..$^{[2]}$

\section{Case report}

\section{Initial presentation}

An 8-day-old infant was brought to the emergency department of a regional hospital in the morning with a history of 14 hours of recurrent seizures. The child's mother had consulted a general practitioner the previous day to look at sores on the child's abdomen. A course of antibiotics, a topical antibiotic and paracetamol were prescribed. Later in the evening, the child suffered seizures. Local traditional practitioners were consulted, to no avail. On arrival at the hospital, the infant was found to have scalding infection of the perineum together with generalised seizures. The following parameters were also recorded: weight $3700 \mathrm{~g}$, temperature $37^{\circ} \mathrm{C}$, length $43 \mathrm{~cm}$, head circumference $35 \mathrm{~cm}$, mid-upper arm circumference $12 \mathrm{~cm}$ and oxygen saturation $98 \%$ in room air. The blood glucose level was normal. Two doses of intravenous (IV) diazepam as well as two doses of IV midazolam were administered but failed to stop the seizures. Appropriate loading doses of valproic acid and phenytoin were given. Eventually a midazolam infusion was started, and after a while no further seizure activity was observed. A lumbar puncture was performed and blood was drawn for various tests (Table 1).

\section{Family background}

The child's 23-year-old mother was married and unemployed. The delivery had been vaginal, the infant weighing $3440 \mathrm{~g}$ and with Apgar scores of 9 and 10 at 1 and 5 minutes, respectively. This was the mother's third pregnancy. The first pregnancy was uneventful, although she had booked late at 6 months. That child is well. She miscarried in the second trimester of the second pregnancy, and a

\begin{tabular}{ll} 
Table 1. Results of laboratory tests done at admission \\
\hline FBC & Normal \\
$\begin{array}{l}\text { Bilirubin (day 5) } \\
\text { U\&E }\end{array}$ & Below phototherapy range (remained low) \\
CMP (mmol/L) & Normal \\
CSF & Normal \\
CRP & 6 mg/L on admission, higher later \\
Pus swab of abdomen & No growth \\
Serum glucose & Normal \\
$\begin{array}{l}\text { FBC = full blood count; U\&E = urea and electrolytes; CMP = calcium, magnesium and } \\
\text { phosphate; CSF = cerebrospinal fluid; CRP = C-reactive protein. }\end{array}$
\end{tabular}

macerated fetus was delivered. During her third pregnancy the mother had booked at the local clinic at 5 months. Two doses of tetanus toxoid were administered. Blood tests were negative for HIV and syphilis. Her blood group was listed as RH-negative, and she was given anti-D immunoglobulin after the baby's birth to prevent rhesus sensitisation.

The husband is 32 years old and works in a coalmine. They live in a shack in an informal settlement in Witbank, Mpumalanga Province. Soon after delivery, the mother went to live with the mother-in-law in a four-roomed house in KwaGuqa township, Witbank. They denied performing any traditional rites on the baby. Surgical spirit was being applied to the umbilical cord stump to prevent infection.

The infant was admitted with the diagnoses of status epilepticus, suspected meningitis and suspected staphylococcal scalded skin syndrome (Fig. 1).

\section{Hospital course}

In the neonatal intensive care unit (NICU), the infant was started on treatment with cefotaxime IV, cloxacillin IV and Bactroban topical antibiotic ointment for the abdomen. After the midazolam infusion was reduced, it was noticed that she responded with spasms to touch, procedures and noise. There were clinical signs suggestive of mild pneumonia. She was observed to be surprisingly mentally alert after prolonged seizures. Besides the spasms she also had a scalded skin infection and a chest infection, but the other systems were normal. 


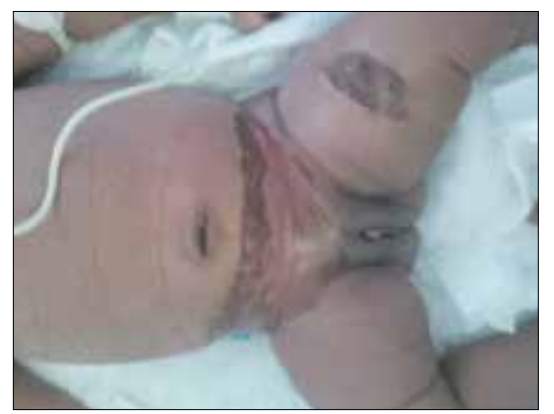

Fig. 1. The distribution of the scalded skin sepsis.

Blood pressure was within normal limits. Occasional sweating was observed. A spatula test was positive. ${ }^{[3]}$ A diagnosis of tetanus was made, and the North West Province Health Department Bana Pele Child Health Handbook ${ }^{[2]}$ protocol was instituted.

After reviewing the above results and making the diagnosis of tetanus, the following treatment was followed:

Wound management. The scalded skin infection areas on the perineum and lower abdomen were debrided, sloughectomy was performed, and the areas were irrigated and dressed with Betadine.

Antibiotics. Metronidazole was administered for 10 days. Penicillin G and IV gentamicin were initiated, but later discontinued in favour of IV cloxacillin.

Supportive management. The infant was ventilated and a maintenance drip was started at $150 \mathrm{~mL} / \mathrm{kg} / \mathrm{d}$. As there were no isolation rooms, screens were used to create a dark, secluded area. Everybody was told to avoid making a noise. Procedures, including gentle suction, were kept to a bare minimum, and sedation was given before these activities. Feeding was started after 2 days. This was well tolerated and increased gradually. The dietician prepared a special diet with extra calories and protein, because tetanus increases daily nutritional requirements. The case was notified.

Sedation. IV phenobarbitone loading was followed by daily oral maintenance. IV chlorpromazine was given (6-hourly) and stopped only when the infant was recovering and experiencing fewer spasms. IV diazepam was used before procedures, as required and when she was paralysed.

Paralysis. IV pancuronium was used, 6-hourly for 2 days, then as required when spasms occurred.

Immunological treatment. The infant was given tetanus immunoglobulin, $250 \mathrm{IU}$ intrathecally and the remaining $250 \mathrm{IU}$ intramuscularly. A second dose of $500 \mathrm{IU}$ was administered intramuscularly.

Vaccination. Tetanus toxoid immunisation was given to both mother and child.
The mother had booked late, so had received only two doses instead of three. Her immunisation history in her previous pregnancies and in childhood is unknown. Antitetanus immunoglobulin levels were not checked before maternal immunisation, in line with accepted cost-control practices. A home visit was done to assess the household and to educate and counsel the family. A very cordial and fruitful meeting was conducted with the grandparents.

After 3 days on IV cloxacillin, the scalded skin showed little improvement. A change was made to IV clindamycin, with good clinical results. The infant was ventilated for 18 days, observed for a further 4 days, and then discharged. She had been free of seizures by day 16 on the ventilator in the NICU.

Standard doses of the above drugs were used. No significant autonomic crises were encountered. The blood pressure remained stable most of the time. The patient developed a Klebsiella nosocomial infection with pneumonia and a C-reactive protein level of $83 \mathrm{mg} / \mathrm{L}$ after 10 days in the NICU. This was sensitive to ciprofloxacin, and she responded well to treatment. There were no significant changes in other blood results during the NICU stay. It is worth noting that our patient, who had significant disease and was treated with intrathecal immunoglobulin, recovered relatively quickly, in 16 days. Intrathecal immunoglobulin has been shown to lead to faster resolution of symptoms compared with intramuscular administration. ${ }^{[4]}$

\section{Discussion \\ Contextual issues}

The context in this case of neonatal tetanus involved the extended family, traditional healers and cultural beliefs. A delay in presentation occurred because the family consulted a healer before bringing the infant to the hospital. The health system, locally and internationally, must continually update its guidelines and practices to try to eliminate this potentially very harmful practice. The extended family mainly involved the grandparents, who are often the custodians of culture and traditions. A home visit with them was scheduled and honoured. The main focus was to educate and demystify the illness, which often starts abruptly and can lead to suspicions of witchcraft. Practices such as applying faecal matter to the cord were discouraged. The family unit was educated about tetanus. (Incidentally, tetanus does not have a universally accepted name in the Sotho and Nguni languages. The Zulu term ngqi, used for tetanus in dictionaries, is not generally known.) The importance of an early visit to the clinic when a neonate is ill was emphasised. Our patient presented only after almost 4 days of a necrotising skin infection, which predisposed her to the tetanus.

The traditional healers involved in the infant's treatment refused to be interviewed, yet such healers are an integral part of the primary care network in SA. It would be of great benefit to the community if these practitioners were to complete the WHOrecommended village health worker's course.

It is essential that the $\mathrm{NDoH}$ maintains the cold chain for vaccines. In our case, investigations are underway to see whether the cold chain had been affected by the recurrent load shedding on the electrical grid. Generators should be available at all sites where vaccines are stored, which is not the case at present. We have seen three cases of neonatal tetanus in our area recently. The $2013 \mathrm{NDoH}$ hospital paediatric tetanus immunisation and treatment guidelines ${ }^{[1]}$ do not cover management of severe cases. The

Table 2. Wound management, tetanus prevention and passive immunity administration

\begin{tabular}{|c|c|c|}
\hline Vaccination status & $\begin{array}{l}\text { Clean, minor wounds including } \\
\text { infection-related ones }\end{array}$ & $\begin{array}{l}\text { All other wounds including } \\
\text { infection-related ones }\end{array}$ \\
\hline $\begin{array}{l}\text { Unknown or }<3 \text { doses } \\
\text { of TT-containing } \\
\text { vaccine }\end{array}$ & $\begin{array}{l}\text { TT and recommend catch-up } \\
\text { vaccination }\end{array}$ & $\begin{array}{l}\text { TT and recommend catch-up } \\
\text { vaccination: TIG }<5 \text { years } 75 \mathrm{IU}, \\
5 \text { - } 10 \text { years } 125 \mathrm{IU},>10 \text { years } 250 \mathrm{IU}\end{array}$ \\
\hline $\begin{array}{l}\geq 3 \text { doses of } \\
\text { TT-containing } \\
\text { vaccine and }<5 \text { years } \\
\text { since last dose }\end{array}$ & No indication & No indication \\
\hline $\begin{array}{l}\geq 3 \text { doses of TT- } \\
\text { containing vaccine } \\
\text { and }>5 \text { years since } \\
\text { last dose }\end{array}$ & TT recommended & TT recommended \\
\hline
\end{tabular}


fact that higher-than-usual diazepam doses are often needed, and are well tolerated, is not stated. This inadequacy should be addressed as a matter of urgency.

The WHO should aim for eradication of tetanus. A country is certified as having eliminated the disease if there is $<1$ case per 1000 live births for at least 2 years. A comprehensive immunisation programme can eradicate neonatal tetanus (zero cases target). This is possible if all pregnant women are fully immunised. Treatment of this condition is expensive, while immunisation is far cheaper. It is time to aim for the eradication target.

\section{Prevention of tetanus in children}

Serum immunoglobulin levels of $>0.01 \mathrm{IU} / \mathrm{mL}$ are protective. Infants should be immunised, as advised by the WHO. In SA, this guideline is offered in the national 'Road To Health' booklet. It is important to administer a booster after every 5 years and to follow antenatal tetanus immunisation protocols. Neonates who have had tetanus should be immunised at discharge, as per the guidelines. However, it is safe to immunise patients at diagnosis. This also avoids the possibility of its being forgotten on the day of discharge.

Table 2 summarises wound management, prevention and passive immunity administration. Our patient contracted tetanus as a result of a complication related to the neonatal scalded skin syndrome. Tetanus immunisation should be considered in neonates with skin infections. Significant skin disruptions, such as epidermolysis bullosa and other severe infectious or vesicular eruptions, must also be considered indications for immunisation (Table 2).

1. National Department of Health, South Africa. Infective and infectious disease. In: Hospital Leve Paediatrics. Standard Treatment Guidelines and Essential Medicines List. Chapter 8, section 36. Pretoria: NDoH, 2013

2. National Department of Health, South Africa. The newborn. In: Bana Pele Child Health Handbook, North West Province. Chapter 3.16. Pretoria: NDoH, 2014:145

3. Apte NM, Karnad DR. The spatula test: A simple bedside test to diagnose tetanus. Am J Trop Med Hyg 1995;53(4):386-387.

4. Ahmad A, Qaisar I, Naeem M, Mazhar AU, Ashfaq M. Intrathecal anti-tetanus human immunoglobulin in the treatment of neonatal tetanus. J Coll Physicians Surg Pak 2011;21(9):539-541.

Accepted 6 June 2016. 